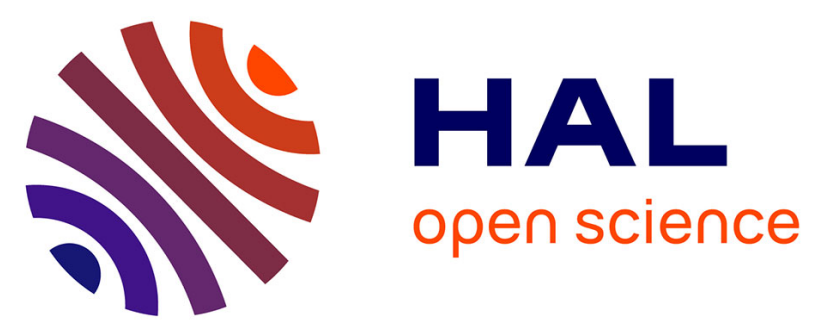

\title{
Ellipsoid-constrained robust fitting of quadrics with application to the 3D morphological characterization of articular surfaces..
}

\author{
Stéphane Allaire, Jean-José Jacq, Valérie Burdin, Christian Roux
}

\section{To cite this version:}

Stéphane Allaire, Jean-José Jacq, Valérie Burdin, Christian Roux. Ellipsoid-constrained robust fitting of quadrics with application to the 3D morphological characterization of articular surfaces... EMBS 2007 : 29th Annual International Conference of the IEEE Engineering in Medicine and Biology Society, Aug 2007, Lyon, France. pp.5087 - 5090, 10.1109/IEMBS.2007.4353484 hal-02164579

\author{
HAL Id: hal-02164579 \\ https://hal.science/hal-02164579
}

Submitted on 25 Jun 2019

HAL is a multi-disciplinary open access archive for the deposit and dissemination of scientific research documents, whether they are published or not. The documents may come from teaching and research institutions in France or abroad, or from public or private research centers.
L'archive ouverte pluridisciplinaire $\mathbf{H A L}$, est destinée au dépôt et à la diffusion de documents scientifiques de niveau recherche, publiés ou non, émanant des établissements d'enseignement et de recherche français ou étrangers, des laboratoires publics ou privés. 


\title{
Ellipsoid-Constrained Robust Fitting of Quadrics with Application to the 3D Morphological Characterization of Articular Surfaces
}

\author{
S. Allaire, J.-J. Jacq, V. Burdin, Member, IEEE, and Ch. Roux, Fellow, IEEE
}

\begin{abstract}
This paper addresses the ellipsoid-type-specified fitting of quadratic surfaces, in the scope of model-based global feature extraction within scattered 3D point clouds. At characterizing articular bone surfaces, the quadrics estimated indicate useful overall-symmetry-related intrinsic centers and axes in joints. A constrained weighted least-squares minimization of algebraic residuals is used, with a robust and bias-corrected metric. With only one quadratic constraint involved, every step produces closed-form eigenvector solutions. To guarantee that an ellipsoid is output, we originally exploit a 2D representation called the Quadric Shape Map (QSM) by carrying out a visual study of the influence of shape constraints. The identified ellipsoid guarantee is needed to extract the center and axes in a wrist joint data stemming from 3D medical images.
\end{abstract}

\section{INTRODUCTION}

$\mathrm{W}$ ORKS on the analysis of articular bone surfaces have mostly focused on local features. Yet, global characteristics accounting for kinematic functionalities, such as centers and axes of joints are also important to the orthopedists. Their location is estimated by fitting specific surface primitives: spheres at the hip and the shoulder, and circular cylinders at the knee (see references in [1]). Most methods still use mere least squares. We have more widely addressed the generic shape characterization based on global symmetries within all articular surfaces [1]. To this end, the use of a common quadratic model has been proposed. Its intention is to extract global features, while discarding topographic details. The estimation method must therefore be robust to tolerate the inherent local inaccuracy of such a simple model. Minimizing an objective function using robust techniques is usually most appropriate; a Hough-transform approach is impracticable at this parameter space dimension.

In this paper we offer the user an option for guaranteeing the specified ellipsoid type even when this type of quadric may seem inadequate with respect to the data, in order to: a) encode morpho-functional knowledge; or b) further ensure a repeatable characterization of complex, pathological or degraded structures so that results are stable and comparable. This does not mean that the ellipsoid is necessarily a sphere.

To this respect, generic non-typologically constrained quadric fitting might be successful, provided the data shape is already close enough to an ellipsoid, such as in the hip [1]. Other techniques including iterative rejection testing may

J.-J. Jacq, V. Burdin and Ch. Roux are with GET-ENST Bretagne, Brest, F-29200 France and INSERM, U650, Brest, F-29200 France. Their former colleague S. Allaire (stephane.allaire@enst-bretagne.fr) is now with the Radiation Medicine Program, Princess Margaret Hospital, Uni. Health Network, 610 University Av., suite 5-612, Toronto, ON M5G 2M9, Canada. help, yet without guarantee either on the type of quadric [2], [7]. Moreover, the objects we wish to characterize are bone surfaces involved in skeletal joints, stemming from natural adaptive anatomical shaping. Thus, no ground truth exists about the suitable quadric primitive. We can make use of the methodological inputs about approximation from computer vision works [2]-[6]. We are nevertheless facing the current challenge of moving on to $3 D$ quadric type constraining.

Throughout the literature, we can delineate several approaches for enforcing specified types of quadric. The tuning of a specialized parameterization for each particular type of quadric can provide guarantee [5], [6]. However, this implies the loss of the closed-form linearity at each step; besides it lacks practical genericity. The only way to keep to direct resolution is to rely on algebraic constraints, through investigating extensions of the innovation that A. Fitzgibbon et al. made to guarantee an ellipse at fitting conic curves to any input $2 \mathrm{D}$ data [3].

In this paper, we describe a method for practically enforcing a constraint that guarantees an ellipsoid among the outputs. The ellipsoid-specific constraint proposed by Q. Li and J.G. Griffiths [8] is revisited. In addition, the constraint blends into the constrained robust fitting framework using Iterative Reweighted Least Squares (IRLS) that we retained [1], as described in section II. It is similar to what was performed in the 2D case of ellipses by R. Halír [4]. Previously, we have proposed a novel bi-dimensional diagram called the Quadric Shape Map (QSM) to analyze and compare the output primitives of the fitting procedure [1]. In section III, the QSM meaningfully justifies the ellipsoid guarantee. The input data is a point cloud, possibly sparse and unevenly scattered. Articular surfaces at the wrist joint get characterized in section IV thanks to the constraint.

\section{METHOD FOR CONSTRAINED RoBUSt QuAdRIC FITTING}

\section{A. Formulation of the Minimization Problem}

In the estimation phase, a quadric is defined in the $3 \mathrm{D}$ space as the zero-level surface of a quadratic polynomial:

$$
f: \boldsymbol{x}(x, y, z) \mapsto f(\boldsymbol{a}, \boldsymbol{x})=\boldsymbol{a}^{\mathrm{T}} \chi,(1)
$$

with the parameter vector $\boldsymbol{a}$ and the basis vector $\chi$ :

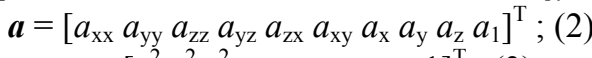

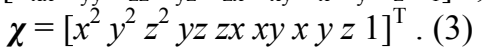

We have retained the implicit representation, making at first no a priori assumption about the shape or type of the sought for quadric (e.g. sphere, cylinder) [1]. For a given cloud of $n$ points $p_{i}=\left[\begin{array}{ll}x_{i} & y_{i} z_{i}\end{array}\right]^{\mathrm{T}}$ and the associated regression vectors $\chi_{i}$, 
the estimate of $\boldsymbol{a}$ is sought for, that defines the most relevant quadric and we can derive its axes, center and semi-axes.

To use a closed-form linear method, we take the squares of the residuals $f\left(\boldsymbol{a}, \boldsymbol{p}_{i}\right)$ as straightforward measures of errors. Yet, the robust norm of a redescending M-estimator discards outliers, and we correct the first order of the bias induced by not using the Euclidean distances. The implied nonlinear minimization is done by the IRLS process, via successive linear steps [1]. Each step minimizes the residual $\chi^{2}$, the weighted scatter matrix $S_{\mathrm{w}}$ being updated from the previous iteration:

$$
\chi^{2}=\sum_{i=1}^{n} w_{i}^{(j-1)} f\left(\boldsymbol{a}, \boldsymbol{p}_{i}\right)^{2}=\boldsymbol{a}^{\mathrm{T}}\left[\sum_{i=1}^{n} w_{i}^{(j-1)} \chi_{i} \chi_{i}^{\mathrm{T}}\right] \boldsymbol{a}=\boldsymbol{a}^{\mathrm{T}} \boldsymbol{S}_{\mathrm{w}} \boldsymbol{a} .
$$

\section{B. Categorization of Constraints}

To avoid the trivial solution $\mathbf{0}_{10}$, the parameter vector $\boldsymbol{a}$ needs to be scaled by a normalization factor. Provided at most one quadratic constraint is enforced, a linear direct closed-form resolution is possible, as described in II.C. It is expressed through a symmetric constraint matrix $\boldsymbol{C}$ :

$$
\boldsymbol{a}^{\mathrm{T}} \boldsymbol{C} \boldsymbol{a}=1 \text {, with } \boldsymbol{C}=\left(\begin{array}{c:c}
\boldsymbol{C}_{\mathrm{q}} & \mathbf{0}_{6 \times 4} \\
\hdashline \mathbf{0}_{4 \times 6} & \mathbf{0}_{4 \times 4}
\end{array}\right) .
$$

Profitably, such a constraint a) is invariant under orientationpreserving isometries of the data and proportional to coordinate scaling since it only involves the shape-related coefficients of quadratic monomials in $f ;$ b) can be given a typological meaning. In the generic case without prior knowledge, we retained a normalizing constraint that bears no singularities among quadrics [1, Eq.7]. In contrast here, the constraint is made typologically specific by aiming at discrimination among the types of quadrics. How we choose the specific nonsingular $6 \times 6$ submatrix $C_{\mathrm{q}}$ is explained in III.

\section{Eigenvector-Based Estimation Technique}

At each direct fitting step, the constrained minimization involves the Lagrangian, with a Lagrange multiplier $\lambda$ :

$$
L(\boldsymbol{a}, \lambda)=\boldsymbol{a}^{\mathrm{T}} \boldsymbol{S}_{\mathrm{w}} \boldsymbol{a}+\lambda\left(1-\boldsymbol{a}^{\mathrm{T}} \boldsymbol{C a}\right)
$$

The necessary condition for first-order optimality reads:

$$
\boldsymbol{S}_{\mathrm{w}} \boldsymbol{a}=\lambda \boldsymbol{C a} \text {. (7) }
$$

Thus a minimizer $(\lambda, \boldsymbol{a})$ must be one of the eigenvalueeigenvector solutions of the generalized eigenvalue problem (7). Besides, note that the residual chi-square (4) is such that:

$$
\chi^{2}=\boldsymbol{a}^{\mathrm{T}} \boldsymbol{S}_{\mathrm{w}} \boldsymbol{a}=\lambda
$$

Using Sylvester's Law of inertia, A.W. Fitzgibbon et al. showed that the generalized eigenvalues of the problem (7) have the same signs as the eigenvalues of the constraint matrix $\boldsymbol{C}$, up to a permutation [3]. Consequently, the solution minimizing (4) subject to the quadratic constraint (5) is the eigenvector $\boldsymbol{a}$ of the problem (7) associated with the eigenvalue $\lambda$ that is simultaneously: a) positive (or equivalently such that $\boldsymbol{a}^{T} \boldsymbol{C a}$ is positive); and b) closest to zero to minimize the residual $\chi^{2}(8)$.

\section{Tools for Building and Understanding Constraints}

Let us now introduce the meaningful expression of the functional (1) as an affine quadratic form:

$$
\begin{gathered}
f: \boldsymbol{x} \mapsto f(\boldsymbol{a}, \boldsymbol{x})=\boldsymbol{x}^{\mathrm{T}} \boldsymbol{\Omega} \boldsymbol{x}+\boldsymbol{l}^{\mathrm{T}} \boldsymbol{x}+k,(9) \\
\text { with: } \boldsymbol{\Omega}=\left[\begin{array}{ccc}
a_{\mathrm{xx}} & \frac{a_{\mathrm{xy}}}{2} & \frac{a_{\mathrm{zx}}}{2} \\
\frac{a_{\mathrm{xy}}}{2} & a_{\mathrm{yy}} & \frac{a_{\mathrm{yz}}}{2} \\
\frac{a_{\mathrm{zx}}}{2} & \frac{a_{\mathrm{yz}}}{2} & a_{\mathrm{zz}}
\end{array}\right], \boldsymbol{l}=\left[\begin{array}{l}
a_{\mathrm{x}} \\
a_{\mathrm{y}} \\
a_{\mathrm{z}}
\end{array}\right] \text { and } k=a_{1} .
\end{gathered}
$$

It can be shown easily that the quadratic form $\boldsymbol{x} \mapsto \boldsymbol{x}^{\mathrm{T}} \boldsymbol{\Omega} \boldsymbol{x}$ will not be affected by orientation-preserving similarities on the quadric defined by $f$, unlike both the linear form $\boldsymbol{x} \mapsto \boldsymbol{l}^{\mathrm{T}} \boldsymbol{x}$ and the constant form $\boldsymbol{x} \mapsto k$. This quadratic form is thus uniquely attached to the quadric considered. Its algebraic reduction involves the eigen decomposition of its symmetric representing matrix $\boldsymbol{\Omega}$, yielding the three all real eigenvalues $\alpha_{i}$ that define the shape of the quadric, and the eigenvectors giving the three axis directions of the quadric.

The coefficients of the characteristic polynomial of $\boldsymbol{\Omega}$ are combinations of these eigenvalues and therefore invariant under changes of direct orthonormal bases. With respect to $\boldsymbol{\Omega}$, they are namely its trace, the sum of its principal secondorder minors $\operatorname{det}_{2}^{\mathrm{P}}(\boldsymbol{\Omega})$, and its determinant:

$$
\begin{gathered}
\alpha_{1}+\alpha_{2}+\alpha_{3}=\operatorname{tr}(\boldsymbol{\Omega})=a_{\mathrm{xx}}+a_{\mathrm{yy}}+a_{\mathrm{zz}} ; \\
\sum_{i \neq j} \alpha_{i} \alpha_{j}=\sum_{i=1}^{3} \operatorname{det}_{2}^{\mathrm{P}}(\boldsymbol{\Omega})=\left|\begin{array}{cc}
a_{y y} & \frac{a_{y z}}{2} \\
\frac{a_{y z}}{2} & a_{z z}
\end{array}\right|+\left|\begin{array}{cc}
a_{z z} & \frac{a_{z x}}{2} \\
\frac{a_{z x}}{2} & a_{x x}
\end{array}\right|+\left|\begin{array}{cc}
a_{x x} & \frac{a_{x y}}{2} \\
\frac{a_{x y}}{2} & a_{y y}
\end{array}\right| ; \\
\alpha_{1} \alpha_{2} \alpha_{3}=\operatorname{det}(\boldsymbol{\Omega}) \text {. (11) }
\end{gathered}
$$

Here we have an invariant of each order with respect to the parameter vector $\boldsymbol{a}$ : linear, quadratic and cubic respectively. They make up a generating basis for all symmetric polynomials of order up to three in the eigenvalues $\alpha_{i}$ of $\boldsymbol{\Omega}$. These are to be involved in specific constraints, since we cannot distinguish the role each eigenvalue of $\boldsymbol{\Omega}$ plays individually.

The Quadric Shape Map (QSM) is a 2D layout locating characteristic zones for the useful types of quadric, and also spotting particular shapes such as ones of revolution [1]. The QSM helpfully allows interpretation in terms of shape continuity and typological transitions, yet it is not to be read linearly. The two coordinate axes $(\beta, \gamma)$ express the relative ratios of two eigenvalues with respect to the numerically highest one for every considered 3D quadric. The QSM is based on these merely unrestrictive conventions:

$$
\alpha_{1} \geq \alpha_{2} \geq \alpha_{3} ; \alpha_{1}>0 \text {, and } \alpha_{2} \geq 0 \text {. (12) }
$$

The ratios are further defined as follows:

$$
\alpha_{2}=\beta \cdot \alpha_{1} \text { and } \alpha_{3}=\gamma \cdot \alpha_{1} \text {, with } 1 \geq \beta \geq 0 \text { and } \beta \geq \gamma \text {. (13) }
$$

Each point in the QSM carries the information of the type and anisotropy of the associated quadrics, which is ideal for designing constraints regardless of pose and scale. It is actually both a quantitative and intuitive representation that is analogous in significance to the 1D continuous scale of eccentricity for 2D conics. By plotting in the QSM the locus of any function of the eigenvalues of $\boldsymbol{\Omega}$ being zero, positive or negative, we can assess its specificity to types of quadrics. 
TABLE I. USEFUL TYPES OF QUADRATIC SURFACES

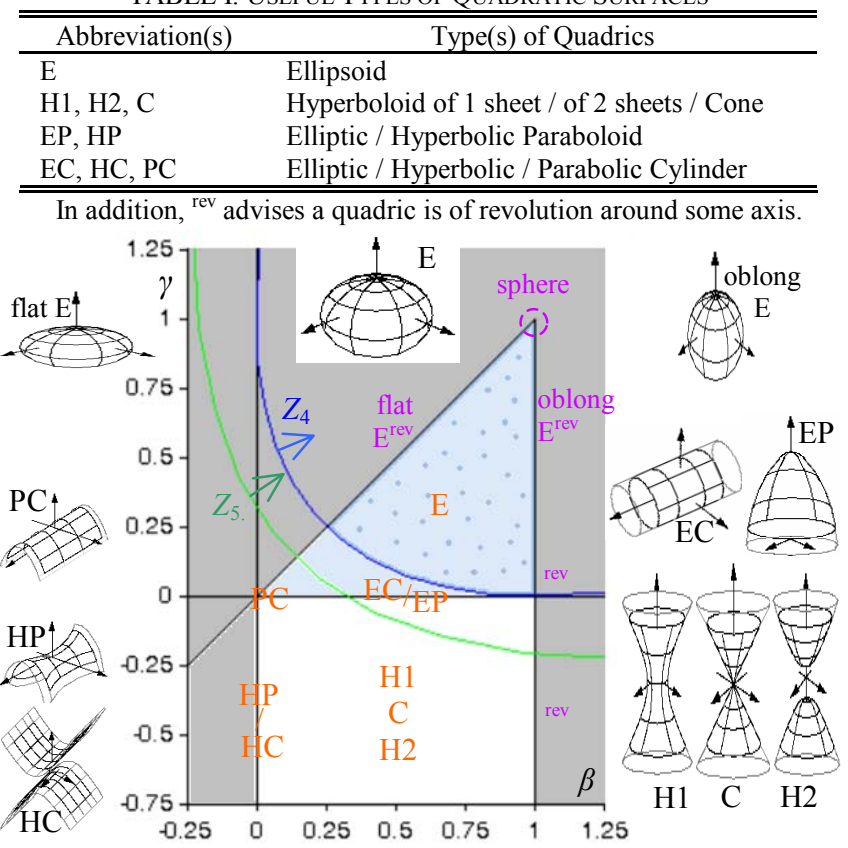

Figure 1. Plots in the Quadric Shape Map (see Table 1) of the inwardpointing zero-level set $Z_{\alpha}$ of constrainable quadratic invariant $c_{\alpha}(\boldsymbol{\Omega})$ (14)

\section{ELLIPSOID-GUARANTEEING CONSTRAINT}

In the $2 \mathrm{D}$ case of conic curves, proper conic types can be discriminated according to the sign of $\operatorname{det}(\boldsymbol{\Omega})$, which is then quadratic with respect to $\boldsymbol{a}$. A. Fitzgibbon $e t$ al. had the idea of merging this inequality constraint with the normalization factor [3]. By enforcing $4 \operatorname{det}(\boldsymbol{\Omega})=+1$, an ellipse solution is guaranteed. The extension of this approach to the 3D case of ellipsoids is not straightforward. The main issue is then the third order of $\operatorname{det}(\boldsymbol{\Omega})$ : for instance, enforcing $\operatorname{det}(\boldsymbol{\Omega})=+1$ falls outside our framework of closed-form estimation. Moreover, the sign of $\operatorname{det}(\boldsymbol{\Omega})$ changes with the undetermined orientation of the normal vectors to the quadric surface (given by the gradient of the functional (9), these can be either outward- or inward-pointing, while parameter vectors $-\boldsymbol{a}$ and $\boldsymbol{a}$ implicitly define the same quadric). Both issues require us to stick to a single quadratic constraint. We have thus devised and investigated a combination parameterized by a scalar $\alpha$ :

$$
c_{\alpha}(\boldsymbol{\Omega})=\alpha \sum \operatorname{det}_{2}^{\mathrm{P}}(\boldsymbol{\Omega})-\operatorname{tr}(\boldsymbol{\Omega})^{2}=1 \text {. (14) }
$$

This quantity was recently considered analytically in [8]. Now, the sign of $c_{\alpha}(\boldsymbol{\Omega})$ actually depends on the coordinates $\beta$ and $\gamma$ via an affine quadratic form of known coefficients:

$$
c_{\alpha}(\boldsymbol{\Omega})=\alpha_{1}^{2} \cdot\left(-\beta^{2}-\gamma^{2}+(\alpha-2)(\beta \gamma+\gamma+\beta)-1\right) \text {. (15) }
$$

In particular, its zero-set $Z_{\alpha}$ is a conic curve in the QSM, which we have geometrically characterized according to the numerical value of $\alpha$. From this detailed examination, we can report that when $\alpha>0$, the conic $Z_{\alpha}$ is always inward-pointing. As $\alpha$ increases, $Z_{\alpha}$ appears in the QSM for $\alpha=3$ as the single point $(1,1)$ associated with the sphere (all eigenvalues of $\boldsymbol{\Omega}$ being equal). Subsequently for $\alpha>3, Z_{\alpha}$ grows as a real ellipse enclosing $(1,1)$, opens up for $\alpha=4$ into the parabola $Z_{4}$ (in Fig.1) and, for $\alpha>4, Z_{\alpha}$ becomes a hyperbola, which gets closer to the origin $(0,0)$ as $\alpha$ further increases towards $+\infty$.

Graphical analysis in the QSM (Fig.1) thus allows us to easily determine whether a given quadric $Q_{0}$ satisfies a constraint on the sign of $c_{\alpha}\left(\boldsymbol{\Omega}_{0}\right)$, by checking on what side of the oriented curve $Z_{\alpha}$ the associated point $\left(\beta_{0}, \gamma_{0}\right)$ is. We have observed that the only curves $Z_{\alpha}$ that can enclose a region within a type-characteristic zone in the QSM are those for $3 \leq \alpha \leq 4$. More precisely, for $3 \leq \alpha \leq 4 \quad c_{\alpha}(\boldsymbol{\Omega})>0$ is a sufficient condition for the quadric to be an ellipsoid, yet not of any shape. Actually, the greater $\alpha$ over 3, the less restraining the constraint $c_{\alpha}(\boldsymbol{\Omega})>0$ is as regards the shape of the guaranteed output ellipsoid. The constraint $c_{4}>0$ is thus the best, but still a subset of ellipsoids remain not included in the guarantee. These are associated with a point located under the curve $Z_{4}$ in the QSM, for instance in the left corner of the ellipsoidzone (then the two eigenvalues $\alpha_{2}$ and $\alpha_{3}$ of $\boldsymbol{\Omega}$ are relatively small with respect to $\alpha_{1}$ ). In terms of shape, the ellipsoids in this latter particular case are rather flat-shaped, while "longthin" or "bar-shaped" ones are not missed, contrary to what is suggested in [8]. (In our ordered convention (12), the geometric minor, medium and major radii of an ellipsoid are proportional to the square roots of the reciprocals of $\alpha_{1}, \alpha_{2}$, $\alpha_{3}$ respectively.) Besides, the QSM workspace helpfully supports all other statements made in [8]. Thus Fig.1 also shows that, as soon as $\alpha>4$, the constraint $c_{\alpha}(\boldsymbol{\Omega})>0$ no longer guarantees an ellipsoid since the curve $Z_{\alpha}$ then encloses other types of quadrics in the QSM (namely H1, H2, C, EP, EC).

In the practical estimation, the chosen constraint $c_{4}(\boldsymbol{\Omega})>0$ is incorporated in the quadratic constraint (5) by setting:

$$
\boldsymbol{C}_{\mathrm{q}}=\left(\begin{array}{ccc:c}
-1 & 1 & 1 & \\
1 & -1 & 1 & \mathbf{0}_{3 \times 3} \\
1 & 1 & -1 & \\
\hdashline & \mathbf{0}_{3 \times 3} & & -\mathbf{I}_{3}
\end{array}\right)
$$

with the $3 \times 3$ identity matrix $\mathbf{I}_{3}$. Since $\boldsymbol{C}_{\mathrm{q}}$ has exactly one single positive eigenvalue (which is simple) and two negative eigenvalues (double and triple, respectively), so does the generalized eigenvalue problem (7) (see II.C). While the single output parameter eigenvector satisfying the constraint $c_{4}(\boldsymbol{\Omega})=1$ assuredly yields an ellipsoid, no guarantee is given about the types of the 5 quadrics implied by the other eigenvectors (satisfying $c_{4}(\boldsymbol{\Omega})=-1$ ). Nearly any non-ellipsoidal quadric might be output, but also flat-shaped ellipsoids. Thus as a matter of fact, at each iterated linear fitting step, this method yields at least one ellipsoid, which by principle may not be the absolutely closest primitive to data in the $\chi^{2}$ sense.

The QSM also gives an intuitive idea of the deformation bias induced on all fitted quadrics by the repulsion away from the set $Z_{4}$ of singularities of the constraint $c_{4}(\boldsymbol{\Omega})=1$. For instance the guaranteed ellipsoid avoids resembling: -elliptic paraboloids and cylinders of revolution associated with the point $(1,0)$ (prolate ellipsoids of revolution are possible fits, as long as they are not too oblong), -the ellipsoid of revolution associated with $(1 / 4,1 / 4)$ flattened at poles, of equatorial radius twice its polar one. The shape of the guaranteed ellipsoid fit thus tends to be rounder towards sphericity (cornered to $(1,1)$ in the QSM) than the point cloud data actually is. 

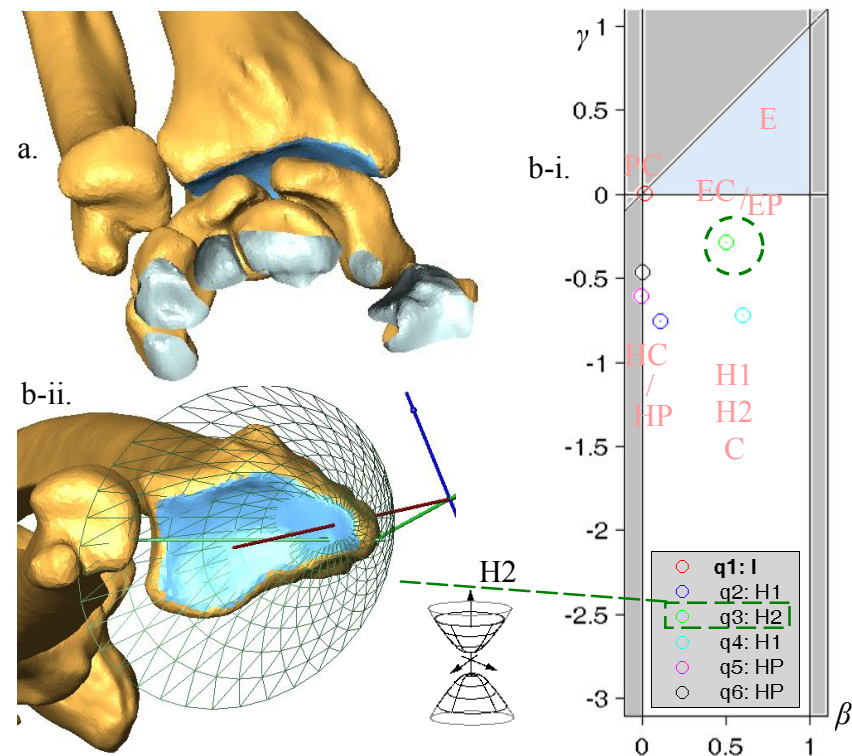

Figure 2. Characterization of the carpal articular surface of the radius bone in the wrist joint: a) input point cloud (blue); b) first direct fit without typological constraint: output eigen quadrics in the QSM (i): q1 is a degenerated line ' 1 '; $\mathrm{q} 3$ is an overall fitting convex sheet of a H2 (ii)

\section{APPLICATION TO THE WRIST JOINT AND DISCUSSION}

We have applied this new type-enforcing scheme to radiocarpal articular surfaces. The input point cloud data stemmed from $\mathrm{CT}$ volume scans. Interactive preprocessing was bone surface segmentation and articular region delimitation (Fig.2a). The radio-carpal joint in the wrist is anatomically classified among condyloid joints (2 degrees of freedom). The characterizing quadric is therefore expected to be an ellipsoid. Yet, no such primitive is output by the generic fitting (Fig.2b: we plotted in the QSM the points associated with the 6 output eigensolutions). The ellipsoid-guaranteeing constraint incorporates prior type knowledge to stably extract the articular axes, center, and dimensions (Fig.3).

From our experience, having processed other healthy articular surfaces of ball-and-socket joints (hip, shoulder, talo-navicular at the rear foot) or ellipsoid joints (humeroradial at the elbow), this ellipsoid-guaranteeing constraint has so far only proved needed for the wrist. However it may become necessary for pathological data, especially for articular surfaces with little extent such as in the shoulder.

As regards assessment, Q. Li and J.G. Griffiths [8] already compared the ellipsoid-specific method with generic ones on synthetic experimental data, yet only considering Gaussian noise level. The constraint effects, such as the rounding bias induced by the clearance of singularities, still need to be further tested on corrupted real data. Like for validating the robustness (excellent repeatability and precision) of the articular center extraction without prior knowledge [1], this should involve experiments against both patches of coherent outliers and upstream intra- and inter-operator variations.

\section{ACKNOWLEDGMENT}

The authors wish to thank C. Lefèvre, D. Colin and G. Moineau (INSERM, U650, Brest; CHU Brest, Service d'Orthopédie Traumatologie; F-29200 France) for providing and interactively processing the input data.

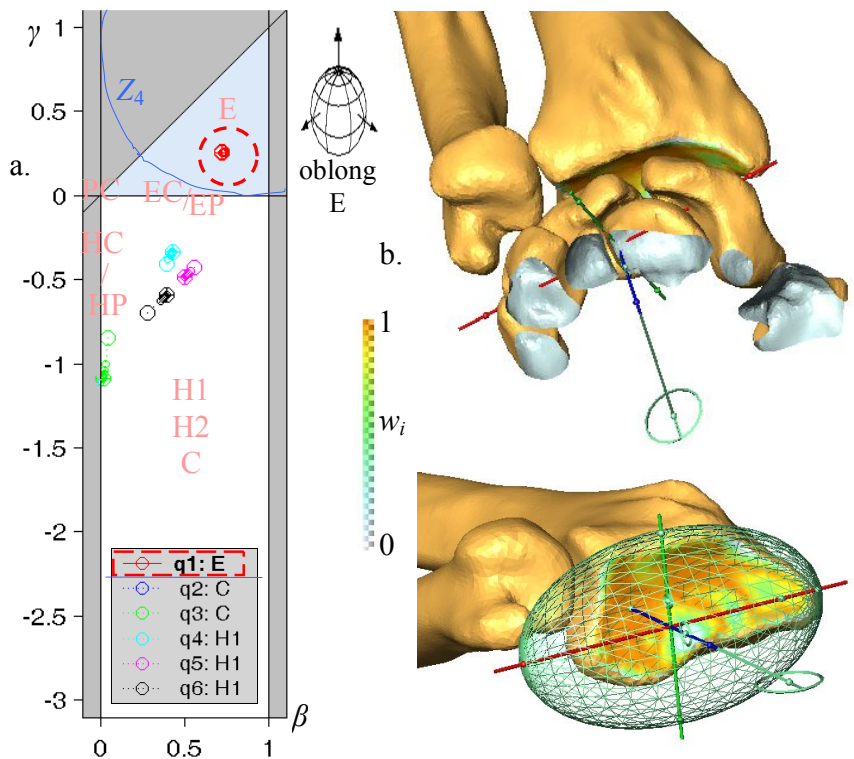

Figure 3. Characterization with prior knowledge through ellipsoidguaranteeing typological constraint (16) in (5): a) eigen solutions depicted in the QSM, refined through robust IRLS; b) the globally fitting output ellipsoid as $1^{\text {st }}$ primitive, providing articular symmetry axes (RGB) intersecting at the symmetry center, extremal curvature radii and centers (green pins); final influence weight map on points (inliers have a hue proportional to fitting goodness; outliers are white).

\section{CONCLUSION}

In this paper, we have presented an ellipsoid-guaranteed robust quadric fitting and applied it to incorporate prior knowledge at globally characterizing the morphology of articular surfaces. The prime global symmetries thus revealed are expected to provide a meaningful insight as regards the functional mobility and stability of a joint, including in the field of feasible prosthesis design and pose [9]. We have exploited the Quadric Shape Map as a convenient $2 \mathrm{D}$ representation to derive and understand a constraint that guarantees an ellipsoid output from any point data.

\section{REFERENCES}

[1] S. Allaire, et al., "Robust Quadric Fitting and Mensuration Comparison in a Mapping Space Applied to 3D Morphological Characterization of Articular Surfaces," in Proc. 4th IEEE ISBI, Washington, April 2007, pp. 972-975.

[2] P.L. Rosin, "A note on the least squares fitting of ellipses," Pattern Recognition Letters, vol. 14, no. 10, pp. 799-808, Oct. 1993.

[3] A.W. Fitzgibbon, M. Pilu, and R.B. Fisher, "Direct Least Square Fitting of Ellipses," IEEE T. PAMI, vol. 21, no. 5, pp. 476-480, 1999.

[4] R. Halír, "Robust bias-corrected least squares fitting of ellipses," in Proc. 8th WSCG, Plzeň, February 2000, vol. I-II.

[5] N. Werghi, R.B. Fisher, C. Robertson, and A.P. Ashbrook, "Modelling Objects Having Quadric Surfaces Incorporating Geometric Constraints," in Proc. 5th ECCV, June 1998, pp. 185-201.

[6] S.J. Ahn, W. Rauh, H.S. Cho, and H.J. Warnecke, "Orthogonal Distance Fitting of Implicit Curves and Surfaces," IEEE Trans. PAMI, vol. 24, no. 5, pp. 620-638, May 2002.

[7] N. Grammalidis, N. Sarris, C. Varzokas, and M.G. Strintzis, "Generation of 3-D Head Models from Multiple Images Using Ellipsoid Approximation for the Rear Part," in Proc. 7th IEEE ICIP, Sept. 2000, vol. 1, pp. 284-287.

[8] Q. Li and J.G. Griffiths, "Least Squares Ellipsoid Specific Fitting," in Proc. IEEE Computer Society GMP, April 2004, pp. 335-340.

[9] N. M. Grosland, "Influence of Articular Geometry on Prosthetic Wrist Stability," CORR, Lippincott, vol. 421, pp. 134-142, 2004. 